\title{
Gastrointestinal ulceration as a possible side effect of bevacizumab which may herald perforation
}

\author{
J. Tol • A. Cats $\cdot$ L. Mol • M. Koopman • \\ M. M. E. M. Bos • J. J. M. van der Hoeven • \\ N. F. Antonini • J. H. J. M. van Krieken • C. J. A. Punt
}

Received: 17 December 2007 / Accepted: 19 February 2008 / Published online: 12 March 2008

(C) The Author(s) 2008

\begin{abstract}
Summary Chemotherapy plus bevacizumab is currently considered as the standard 1st line treatment of advanced colorectal cancer (ACC). Whereas GI perforation is a known side effect of bevacizumab, the development of GI ulcers has not been reported. We identified 18 patients with ACC who participated in a phase III multicentre trial which included chemotherapy and bevacizumab, who developed a GI ulcer $(n=6)$, perforation $(n=8)$ or both $(n=4)$. The risk
\end{abstract}

J. Tol · M. Koopman · C. J. A. Punt $(\bowtie)$

Department of Medical Oncology,

Radboud University Nijmegen Medical Centre,

P.O. Box 9101, 6500 HB Nijmegen, The Netherlands

e-mail: C.Punt@onco.umcn.nl

\section{A. Cats}

Department of Gastroenterology,

The Netherlands Cancer Institute,

Amsterdam, The Netherlands

L. Mol

Comprehensive Cancer Centre East (IKO),

Nijmegen, The Netherlands

M. M. E. M. Bos

Department of Internal Medicine, Reinier de Graaf Gasthuis, Delft, The Netherlands

\section{J. J. M. van der Hoeven}

Department of Internal Medicine, Alkmaar Medical Centre,

Alkmaar, The Netherlands

\section{N. F. Antonini}

Department of Biometrics, The Netherlands Cancer Institute,

Amsterdam, The Netherlands

J. H. J. M. van Krieken

Department of Pathology,

Radboud University Nijmegen Medical Centre,

Nijmegen, The Netherlands of developing a symptomatic GI ulcer or perforation was $1.3 \%$ and $1.6 \%$, respectively. Central review of the histology specimens showed ulceration and/or granulation tissue with neovascularisation. The majority (89\%) of events developed early during treatment. Given these observations, as well as the relationship between VEGF and mucosal injury healing, we suggest that GI ulcers may occur as a side effect of treatment with bevacizumab and may herald perforation.

Keywords Bevacizumab · Perforation .

Gastrointestinal ulcer $\cdot$ Colorectal cancer

\section{Introduction}

Colorectal cancer is the second most common cause of cancer deaths worldwide with 492,000 related deaths in 2000 [1]. Approximately 50\% of patients will eventually develop distant metastases, for which usually palliative systemic treatment is administered. Over the past decades the treatment options for patients with advanced colorectal cancer (ACC) have changed considerably. Currently available cytotoxic drugs, the fluoropyrimidines (5FU, UFT and capecitabine), oxaliplatin and irinotecan have improved the median overall survival from 8 months with supportive care alone to approximately 17-19 months [2]. Recently, a new class of agents has been developed that inhibits signal transduction pathways, and which are usually referred to as targeted therapy. In ACC two drugs of this class are currently being used in the clinic: inhibitors of the epidermal growth factor receptor (EGFR) and of the vascular endothelial growth factor (VEGF) [3]. VEGF is a potent angiogenic factor that exerts its effect by binding to one or more of the VEGF receptors (VEGFR), leading to 
activation of several intracellular signal transduction pathways. In addition to the stimulation of neoangiogenesis, VEGF shows significant mitogenic activity for arterial, venous and lymphatic endothelial cells and plays a role in endothelial cell proliferation, vascular permeability and the inhibition of apoptosis [4]. Bevacizumab (Avastin ${ }^{\circledR}$ ) is a humanized monoclonal antibody to VEGF. The addition of bevacizumab to fluoropyrimidine-containing chemotherapy significantly prolongs the median overall survival (OS) in previously untreated patients with ACC $[5,6]$ and is currently considered as the standard first-line treatment. In 2nd line, bevacizumab also shows a significant increase in OS when added to chemotherapy [7]. Bevacizumab is generally well tolerated. The most common toxicities are hypertension and proteinuria. Arterial thromboembolic events, bleeding diathesis (mainly gastrointestinal bleeding) and gastrointestinal perforations have consistently been reported but at a low frequency. The focus of bleeding has been located along the entire digestive tract. In patients with ACC with their primary tumour in situ and who were treated with chemotherapy plus bevacizumab, the primary tumour was the focus of bleeding in $50 \%(8 / 16)$ of the patients [8]. The overall incidence of GI perforation in large series of ACC patients is approximately $1-2 \%[6,8,9]$. In phase II studies with bevacizumab and chemotherapy the incidence of GI perforations was $6 \%$ in 47 patients with advanced gastric or gastroesophageal junction carcinoma [10], and $8 \%$ in 52 patients with advanced pancreatic cancer [11]. In the latter study, one patient developed ulceration at the site of the gastrojejunostomy anastomosis with secondary perforation at this site. In advanced ovarian cancer, a large variation in the incidence of GI perforations after treatment with bevacizumab either as monotherapy or combined with chemotherapy has been reported, ranging from $0 \%$ to $15.4 \%$ [12]. The intra-abdominal spread of ovarian cancer metastases compared to other cancer types may play a role in the higher incidence of perforations in this disease. The etiology of GI perforations occurring upon treatment with bevacizumab is unknown. Perforations may develop at the site of the primary tumour or elsewhere in the digestive tract, amongst others in the stomach or duodenum [9]. In patients without previous resection of the primary tumour, the site of perforation is related to the primary tumour site in $50 \%$ of the patients [9]. Predisposing factors for GI perforation are acute diverticulitis, GI obstruction, primary tumour in situ, abdominal carcinomatosis and prior abdominal or pelvic radiotherapy [13].

Here we present a series of patients who developed a symptomatic GI ulcer, perforation, or both during treatment with bevacizumab plus chemotherapy in a prospective phase III study. We postulate that GI ulcers are an adverse effect of treatment with bevacizumab and may herald perforation.

\section{Patients and methods}

Patients with ACC were treated within a multicentre phase III study (CAIRO2, CKTO 2005-02) of the Dutch Colorectal Cancer Group (DCCG), and were randomized between capecitabine $1000 \mathrm{mg} / \mathrm{m}^{2}$ orally twice daily day 1-14, oxaliplatin $130 \mathrm{mg} / \mathrm{m}^{2}$ i.v. day 1 , and bevacizumab $7.5 \mathrm{mg} / \mathrm{kg}$ i.v. day 1 (arm A), and the same treatment but with the addition of weekly cetuximab (a chimaeric antibody against the epidermal growth factor receptor) $400 \mathrm{mg} / \mathrm{m}^{2}$ i.v. in week 1 and $250 \mathrm{mg} / \mathrm{m}^{2}$ i.v. weekly thereafter (arm B) [14]. All cycles were given q 3 weeks. Inclusion criteria included a histologically proven ACC with irresectable distant metastases, the presence of at least one measurable disease parameter, WHO performance status 0 or 1 , and adequate organ functions. Main exclusion criteria were prior chemotherapy for advanced disease, bleeding diathesis or coagulation disorders, uncontrolled hypertension, a serious non-healing wound or ulcer, and major surgery within 28 days prior to the start of treatment. Accrual of 755 patients was completed in December 2006. During follow-up we observed several cases of symptomatic GI ulcers and perforations. GI ulcers without perforation were identified during evaluation of abdominal pain or GI bleeding. To investigate a possible relationship between these events, all relevant clinical data of these patients were collected. Available tissue material of ulcers or perforations was centrally reviewed by a pathologist (JvK).

\section{Results}

Eighteen patients presented with a symptomatic GI ulcer (Fig. 1) $(n=6)$, perforation $(n=8)$ or a perforated ulcer $(n=4$; Table 1). Patient characteristics are shown in Table 2. Median age was 64 years (range 50-73). The incidence of GI ulcer, perforation, or perforated ulcer development in the present study was $0.8 \%, 1.0 \%$ and $0.5 \%$ respectively. The overall risk of the development of an ulcer with or without perforation, or a perforation with or without an ulcer was $1.3 \%$ and $1.6 \%$, respectively. The incidence of events was higher in the cetuximab treatment arm (ulcers: two versus four; perforation: five versus three; perforated ulcers: three versus one, respectively). However the small difference in a low number of events does not allow further conclusions. Four ulcers developed in the upper digestive tract and two ulcers developed in the lower digestive tract, of which one at the site of anastomosis at 5 months after colon resection. All eight perforations were located in the lower digestive tract, of which six at the site of the primary tumour or local recurrence. One perforated ulcer developed in the duodenum, two at the site of primary anastomosis 16 months and 3 years after rectum resection, respectively, and one patient devel- 
Table 1 Characteristics of the event

\begin{tabular}{clcll}
\hline Patients & Ulcer/perforation & $\begin{array}{c}\text { Number of } \\
\text { cycles received }\end{array}$ & Site of event & $\begin{array}{l}\text { Relationship } \\
\text { with tumour }\end{array}$ \\
\hline 1 & U & 4 & Stomach & No \\
2 & $\mathrm{U}$ & 2 & Duodenum & No \\
3 & $\mathrm{U}$ & 4 & Colon & $\mathrm{A}$ \\
4 & $\mathrm{U}$ & 12 & Stomach & No \\
5 & $\mathrm{U}$ & 1 & Ileum & No \\
6 & $\mathrm{U}$ & 5 & Stomach & No \\
7 & $\mathrm{U}+\mathrm{P}$ & 3 & Colon & A \\
8 & $\mathrm{U}+\mathrm{P}$ & 2 & Duodenum & No \\
9 & $\mathrm{U}+\mathrm{P}$ & 3 & Colon & A \\
10 & $\mathrm{U}+\mathrm{P}$ & 1 & Duodenum, small intestine, colon & Yes \\
11 & $\mathrm{P}$ & 4 & Colon & Yes \\
12 & $\mathrm{P}$ & 3 & Ileum & A \\
13 & $\mathrm{P}$ & 11 & Colon & Yes \\
14 & $\mathrm{P}$ & 1 & Colon & Yes \\
15 & $\mathrm{P}$ & 4 & Colon/duodenum & Yes \\
16 & $\mathrm{P}$ & 1 & Colon & Yes \\
17 & $\mathrm{P}$ & 2 & Colon & Yes \\
18 & $\mathrm{P}$ & 2 & Colon & No \\
\hline
\end{tabular}

oped multiple ulcers, perforations and perforated ulcers along the entire digestive tract. Both patients who developed a perforated ulcer at the site of the anastomosis following anterior resection previously underwent preoperative radiotherapy on the pelvic region. Sixteen (89\%) events (five ulcers, seven perforations and all four perforated ulcers) developed within the first 15 weeks after the start of treatment. The histological diagnosis was confirmed upon pathologic review in all 13 patients of whom tissue specimens were available (five ulcers, four perforations and all four perforated ulcers). One tumour showed deep ulceration with necrosis at the side of perforation (patient 15). Since no tissue was available from 4 perforations, the presence of ulceration surrounding the perforation could not be assessed in these patients. As bevacizumab is an inhibitor of angiogenesis, and as we presumed that the inhibition of angiogenesis was related to the development of ulcers and perforations, special attention was focussed at the blood vessels surrounding the ulcer or perforation. No special features were seen and the expected neovascularisation

Table 2 Patient characteristics

\begin{tabular}{|c|c|c|c|c|c|c|c|}
\hline Patient & Sex & Age & Treatment arm & Site of primary & Primary in situ & Relevant comedication & Prior radiotherapy on pelvis \\
\hline 1 & M & 58 & A & $\mathrm{C}$ & - & - & - \\
\hline 2 & M & 68 & A & $\mathrm{C}$ & + & PPI, NSAID & - \\
\hline 3 & M & 57 & A & $\mathrm{R}$ & - & PPI, S & - \\
\hline 4 & M & 66 & $\mathrm{~B}$ & RS & + & - & - \\
\hline 5 & M & 62 & A & $\mathrm{C}$ & - & PPI,NSAID & - \\
\hline 6 & M & 66 & $\mathrm{~B}$ & $\mathrm{C}$ & + & - & - \\
\hline 7 & M & 50 & $\mathrm{~B}$ & $\mathrm{R}$ & - & PPI, NSAID & + \\
\hline 8 & $\mathrm{~F}$ & 73 & $\mathrm{~B}$ & $\mathrm{C}$ & - & PPI & - \\
\hline 9 & M & 71 & A & $\mathrm{R}$ & - & PPI, S & + \\
\hline 10 & $\mathrm{~F}$ & 73 & $\mathrm{~B}$ & $\mathrm{C}$ & - & PPI, NSAID & - \\
\hline 11 & $\mathrm{~F}$ & 57 & B & $\mathrm{C}$ & + & PPI & - \\
\hline 12 & M & 60 & $\mathrm{~B}$ & $\mathrm{R}$ & - & - & - \\
\hline 13 & M & 58 & A & $\mathrm{C}$ & + & - & - \\
\hline 14 & M & 72 & $\mathrm{~A}$ & $\mathrm{C}$ & + & NSAID, S & - \\
\hline 15 & M & 67 & $\mathrm{~B}$ & $\mathrm{C}$ & - & - & - \\
\hline 16 & M & 57 & $\mathrm{~A}$ & $\mathrm{C}$ & + & NSAID & - \\
\hline 17 & M & 53 & $\mathrm{~B}$ & $\mathrm{C}$ & + & NSAID & - \\
\hline 18 & $\mathrm{~F}$ & 67 & B & $\mathrm{R}$ & - & - & + \\
\hline
\end{tabular}

Treatment arm A: capecitabine, oxaliplatin, bevacizumab; treatment arm B: capecitabine, oxaliplatin, bevacizumab and cetuximab $M$ Male, $F$ female, $C$ colon, $R$ rectum, $R S$ rectosigmoid, $P P I$ protonpump inhibitor, NSAID non steroidal anti-inflammatory drug, $S$ steroid 
which accompanies ulceration was present. Additional CD68 immunostaining for macrophages (performed on tissue of 10 patients), CD31 and CD34 for assessing vascularity (one patient) and CMV staining (three patients) did not reveal any abnormalities. No Helicobacter pylori colonisation was found in the gastric and duodenal biopsies by histology. In all five patients developing a perforation with a primary tumour in situ, the perforation was located within the primary tumour. Seven perforations occurred in patients after resection of the primary tumour $(58 \%)$, in whom the anastomosis was the site of perforation in three patients. In order to assess other known risk factors for ulceration, the use of relevant comedication (protonpump inhibitors (PPI), non-steroidal anti-inflammatory drugs (NSAID) and steroids) was recorded. Out of seven patients with localisation of an ulcer or perforation in the upper abdominal tract, two patients used NSAID in combination with prophylactic use of PPI at the time of randomisation and a third patient used PPI for unknown indication. Three patients used oral steroids at the time of randomisation.

\section{Discussion}

We observed a symptomatic GI ulcer in 10 patients with ACC (1.3\%) who were included in a phase III study that involved a total of 755 patients treated with chemotherapy and bevacizumab with or without cetuximab. Pathologic review of the ulcers did not show unusual findings. This incidence is higher than the $0.1 \%$ that has been reported for the general population [15]. In four of these patients a perforated ulcer was diagnosed. Since GI perforation is a known side effect of bevacizumab $[6,8,9]$, and ulcers have not been reported as a complication of any systemic anticancer treatment, these findings prompted us to assess the possible relationship between ulcer development and treatment with bevacizumab, as well as between ulcer development and perforation.

Several preclinical studies have been performed on the role of VEGF in GI mucosa and ulcer healing. Neoangiogenesis in general, and VEGF in particular, play an important role in the healing of GI ulcers [16]. In animal models injection of plasmid-DNA encoding VEGF stimulates the healing of gastric ulcers [17]. Moreover, the level of VEGF expression correlates with the size and depth of stress-induced gastric ulcers in murine models. Higher levels of VEGF expression are associated with a decrease in ulcer size and depth [18]. Infusion of an anti-VEGF antibody in rats results in a significantly delayed healing of gastric erosions [19]. Lastly, the inhibitory effect of bevacizumab on wound healing is well established [20], and a non-significant trend for an increased incidence of wound healing complications has been observed during bevacizumab treatment [21]. These data support a role for anti-VEGF therapy in ulcer development.

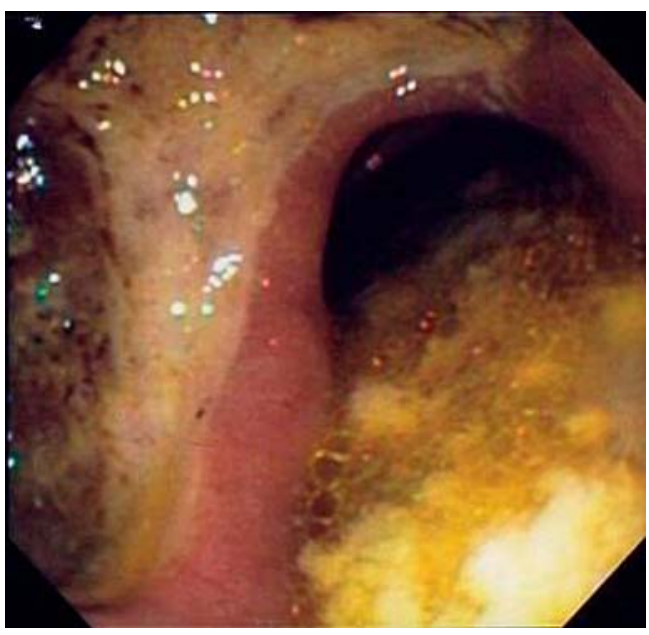

Fig. 1 Gastric ulcer at endoscopy in patient 1

The incidence of perforation in our study was comparable to earlier published data $[6,8,9]$. In four out of 12 patients who presented with a perforation an ulcer was demonstrated at the site of perforation. It should be noted that tissue from perforated sites was only available from eight patients. Furthermore, ulceration at the site of a primary tumour may be a non-specific finding as ulceration and deep necrosis are typical features in malignancies. The aetiology of perforations during bevacizumab treatment is unknown. Since the majority of perforations has been observed at the site of the primary tumour, mucosal injury may be considered as a predisposing factor. In patients with a resected primary tumour, mucosal damage is present at the site of anastomosis. We observed one ulcer, two perforated ulcers and one perforation at the site of anastomosis after bowel resection.

Taken together, these data suggest a relationship between mucosal injury and the development of GI ulceration or perforation. It has been postulated that the development of GI perforations in patients treated with bevacizumab might be the result of mesenteric ischaemia due to the cholesterol emboli syndrome [21]. Since ulceration is a well-known feature of mesenteric ischaemia, this is in agreement with our hypothesis.

NSAID use has been associated with a risk of GI ulceration, which is not limited to the upper GI tract [22]. Six patients with a distal GI ulcer or perforation used NSAID and three patients used steroids. However, a causal relationship is unlikely considering the frequent use of these drugs and the low incidence of symptomatic distal GI ulcers in the general population. Further pathological studies of perforated sites are warranted to confirm ulceration as a predisposing factor for perforation. Of note, a perforated ulcer at the site of anastomosis during treatment with bevacizumab has been reported in a patient with pancreatic cancer [11]. 
The fact that the majority of events in our series occurred early during treatment is consistent with observations in GI perforations by Sugrue et al. [13]. This early occurrence also supports a causal relationship between the development of ulcers and the use of bevacizumab.

In conclusion, we identified symptomatic GI ulcers as a possible side effect of treatment with bevacizumab in patients with ACC. This is supported by the relatively high incidence of ulcers in our study population, the early occurrence of ulcers during bevacizumab treatment, the important role of VEGF in ulcer and mucosal injury healing and the inhibitory effect of bevacizumab on wound healing. We suggest that GI ulcers may herald perforation. Preexistent mucosal lesions may be preferential localisations for the development of ulcers as well as perforations. We recommend performing endoscopic evaluation in any patient during treatment with bevacizumab upon presentation with symptoms that may be related to a GI ulcer, and to closely examine tissue specimens from perforated sites for the presence of ulcers. When further studies confirm the relationship between ulcer development and perforation, patients should be carefully monitored for signs or symptoms related to ulcers, and should an ulcer be diagnosed the administration of bevacizumab should be permanently discontinued.

Acknowledgements The DCCG received grant support from the Commissie Klinisch Toegepast Onderzoek (CKTO) of the Dutch Cancer Foundation (KWF), and unrestricted scientific grants from Merck Serono, Roche and Sanofi-Aventis.

Conflict of interest statement CJA Punt has received research grants and honoraria from Sanofi-Aventis, Roche, and Pfizer. All other authors declare that they have no conflict of interest.

Open Access This article is distributed under the terms of the Creative Commons Attribution Noncommercial License which permits any noncommercial use, distribution, and reproduction in any medium, provided the original author(s) and source are credited.

\section{References}

1. Weitz J, Koch M, Debus J, Hohler T, Galle PR, Buchler MW (2005) Colorectal cancer. Lancet 365(9454):153-165

2. Punt CJ (2004) New options and old dilemmas in the treatment of patients with advanced colorectal cancer. Ann Oncol 15 (10):1453-1459

3. Tol J, Punt CJA (2007) Targeted therapy in advanced colorectal cancer, an update. Targeted Oncology 2(3):165-172 July 2007

4. Ferrara N, Gerber HP, LeCouter J (2003) The biology of VEGF and its receptors. Nat Med 9(6):669-676

5. Kabbinavar FF, Hambleton J, Mass RD, Hurwitz HI, Bergsland E, Sarkar S (2005) Combined analysis of efficacy: the addition of bevacizumab to fluorouracil/leucovorin improves survival for patients with metastatic colorectal cancer. J Clin Oncol 23(16): 3706-3712

6. Hurwitz H, Fehrenbacher L, Novotny W et al (2004) Bevacizumab plus irinotecan, fluorouracil, and leucovorin for metastatic colorectal cancer. N Engl J Med 350(23):2335-2342

7. Giantonio BJ, Catalano PJ, Meropol NJ et al (2007) Bevacizumab in combination with oxaliplatin, fluorouracil, and leucovorin (FOLFOX4) for previously treated metastatic colorectal cancer: results from the Eastern Cooperative Oncology Group Study E3200. J Clin Oncol 25(12):1539-1544

8. Saif MW, Elfiky A, Salem RR (2007) Gastrointestinal perforation due to bevacizumab in colorectal cancer. Ann Surg Oncol 14(6): $1860-1869$

9. Kretzschmar A, Cunningham D, Berry S et al (2006) Incidence of gastrointestinal perforations and bleeding in patients starting bevacizumab treatment in first-line metastatic CRC without primary tumour resection-preliminary results from BEAT [abstract 248]. Proc Am Soc Clin Oncol GI 209

10. Shah MA, Ramanathan RK, Ilson DH et al (2006) Multicenter phase II study of irinotecan, cisplatin, and bevacizumab in patients with metastatic gastric or gastroesophageal junction adenocarcinoma. J Clin Oncol 24(33):5201-5206

11. Kindler HL, Friberg G, Singh DA et al (2005) Phase II trial of bevacizumab plus gemcitabine in patients with advanced pancreatic cancer. J Clin Oncol 23(31):8033-8040

12. Han ES, Monk BJ (2007) What is the risk of bowel perforation associated with bevacizumab therapy in ovarian cancer? Gynecol Oncol 105(1):3-6

13. Sugrue M, Kozloff M, Hainsworth J et al (2006) Risk factors for gastrointestinal perforations in patients with metastatic colorectal cancer receiving bevacizumab plus chemotherapy [abstract 3535]. Proc Am Soc Clin Oncol 24:154S

14. Tol J, Koopman M, Rodenburg CJ et al (2008) A randomised phase III study on capecitabine, oxaliplatin and bevacizumab with or without cetuximab in first-line advanced colorectal cancer, the CAIRO 2 study of the Dutch Colorectal Cancer Group (DCCG). An interim anaylsis of toxicity. Ann Oncol Feb 13 (in press)

15. Garcia Rodriguez LA, Hernandez-Diaz S (2004) Risk of uncomplicated peptic ulcer among users of aspirin and nonaspirin nonsteroidal antiinflammatory drugs. Am J Epidemiol 159(1): 23-31

16. Tarnawski AS (2005) Cellular and molecular mechanisms of gastrointestinal ulcer healing. Dig Dis Sci 50(Suppl 1):S24-S33

17. Gyires K (2005) Gastric mucosal protection: from prostaglandins to gene-therapy. Curr Med Chem 12(2):203-215

18. Malara B, Josko J, Tyrpien M, Malara P, Steplewska K (2005) Dynamics of changes in vascular endothelial growth factor (VEGF) expression and angiogenesis in stress-induced gastric ulceration in rats. J Physiol Pharmacol 56(2):259-271

19. Yoshida M, Wakabayashi G, Ishikawa $H$ et al (2003) A possible defensive mechanism in the basal region of gastric mucosa and the healing of erosions. Clin Hemorheol Microcirc 29(3-4): $301-312$

20. Thornton AD, Ravn P, Winslet M, Chester K (2006) Angiogenesis inhibition with bevacizumab and the surgical management of colorectal cancer. Br J Surg 93(12):1456-1463

21. Mir O, Mouthon L, Alexandre J et al (2007) Bevacizumabinduced cardiovascular events: a consequence of cholesterol emboli syndrome? J Natl Cancer Inst 99(1):85-86

22. Buchman AL, Schwartz MR (1996) Colonic ulceration associated with the systemic use of nonsteroidal antiinflammatory medication. J Clin Gastroenterol 22(3):224-226 